\title{
Commentary on: Suburethral sling procedures in the United States: complications, readmission, and reoperation
}

\author{
Tamara Grisales ${ }^{1}$ (D)
}

Received: 12 September 2017 / Accepted: 12 September 2017 /Published online: 18 October 2017

(C) The International Urogynecological Association 2017

The authors of this cohort study [1] utilized the American College of Surgeons National Safety and Quality Improvement Project (ACS NSQIP) database to evaluate complications, readmissions, and reoperations following suburethral sling procedures performed in the USA from 2012 to 2014. The primary outcome of the study was 30-day hospital readmissions. Secondary outcomes included postoperative complications and reoperations. Female patients were selected using the Current Procedural Terminology (CPT) code for sling procedure for stress urinary incontinence (57288), which may include synthetic midurethral slings as well as pubovaginal slings. Patients undergoing other procedures were excluded.

A total of 7,117 sling procedures were identified during the study period. Demographic data on this cohort of patients were collected: Of those reporting race, $92 \%$ were white, $75.3 \%$ were overweight or obese, and $61.1 \%$ were between the ages of 50 and 70 years, $38.9 \%$ were under the age 50 years, and $13 \%$ were over the age of 70 years. Of the 7,117 procedures, $53 \%$ were performed by gynecologists and $45.8 \%$ by urologists. Nearly all cases $(82.7 \%)$ were performed after general anesthesia, and $87.1 \%$ of the patients were discharged home the same day.

The rate of readmission was $1.16 \%(83 / 7,117)$. Of the 83 readmissions, 44 (53\%) were related to the sling operation, and the most common indications were urinary tract infection (UTI), pneumonia, and surgical site infection. Age, smoking,

Tamara Grisales

TGrisales@mednet.ucla.edu

1 UCLA Medical Center, Los Angeles, CA, USA congestive heart failure, and bleeding disorders, as well as increased American Society of Anesthesiologists score and worse functional status, were all associated with readmission. The rate of postoperative complications was $2.9 \%$, most of which were UTIs. The 30-day reoperation rate was $0.75 \%$ (54/ $7,117)$. The most common indication for reoperation was urinary retention (11 patients, 26.2\%) followed by bleeding complications (6 patients, 14.3\%).

The study highlights the safety of suburethral slings by demonstrating low rates of readmission, complications, and reoperations in the 30-day postoperative period. However, the study likely underestimates the rates of other complications that occur beyond the 30-day follow-up period, such as voiding dysfunction, recurrent infections, pain/dyspareunia, and mesh erosions or exposures. Because such complications are typically identified more than 30 days after sling placement, this study likely also underestimates the reoperation rate for suburethral slings.

While the ACS NSQIP is a large database with surgeryspecific data points, it is limited in its ability to capture only intraoperative and 30-day postoperative data points. Additionally, the CPT code queried includes all suburethral sling procedures including synthetic midurethral slings procedures, as well as pubovaginal and fascial sling procedures, which may also affect the generalizability of the conclusions. Despite its limitations, this cohort study demonstrates low 30day complication, readmission, and reoperation rates.

\section{References}

Propst K, O’Sullivan DM, Tulikangas PK. Suburethral sling procedures in the United States: complications, readmission, and reoperation". Int Urogynecol J. 2017. https://doi.org/10.1007/s00192-017-3293-8. 